\title{
Alpha budgeting - Cross-sectional dispersion decomposed
}

Received (in revised form): 1st February, 2007

\section{Wallace Yu}

is Vice President in Global Markets Equity at Deutsche Bank AG London. He holds a Master's in Mathematical Finance from Imperial College, London (2001) and is a CFA charterholder. This paper was co-authored when he was at Morgan Stanley's Quantitative and Derivative Strategies group.

\section{Yazid M. Sharaiha*}

is Managing Director at Morgan Stanley where he heads the Quantitative and Derivative Strategies group. He holds a Master's in Engineering from UC Berkeley and a PhD in Operations Research from Imperial College, London (1991). He has co-authored one book and published over 30 articles in the fields of optimisation and financial modelling.

*25 Cabot Square, London E14 4QA, UK.

E-mail: yazid.sharaiha@morganstanley.com

Abstract (All work in this paper is based on research conducted by the two authors working for Morgan Stanley and the findings have appeared in the Morgan Stanley Quantitative and Derivative Strategies publication as of March 2006). The last three years have witnessed markedly lower volatility in global equity markets, combined with higher correlation and lower dispersion. This has meant greater challenges for asset allocation and stock selection alpha opportunities appear limited. How should the investment process be structured to maximise exploitation of the available alpha? We introduce cross-sectional standard deviation (or dispersion) as a metric for alpha opportunities, and show how it can shed light on the management of research resources, and on the optimal combination of investment strategies. The metric allows for objective alpha budgeting in the investment process. This involves both the identification of the key top-down drivers of dispersion in a given market regime, and the appropriate 'alpha granularity', which is linked to the optimal concentration of stocks in the portfolio. Alpha budgeting from top-down allocations - country, sector, size, style and beyond: using a novel decomposition framework of cross-sectional dispersion, we show the relative magnitude of top-down allocation decisions for several organisational structures. In Europe, sector investing continues to provide greater opportunities than country investing. Alpha opportunities and volatility - hedging opportunities: we establish a linkage between cross-sectional standard deviation (dispersion) and time-series correlation and volatility. Low return dispersion can be a business model risk for active stock pickers; we show how targeted exposure to volatility can be used as a hedge.

Journal of Asset Management (2007) 8, 58-72. doi:10.1057/palgrave.jam.2250060

Keywords: cross-sectional volatility, dispersion, alpha budgeting, risk decomposition, portfolio construction

\section{Introduction}

The last three years have witnessed markedly lower volatility in global equity markets, combined with higher correlation and lower dispersion. This has meant greater challenges for asset allocation and stock selection - alpha opportunities appear limited. How should the investment process be structured to maximise exploitation of the available alpha?

In this paper we argue that, in addition to setting risk and return targets, understanding the current 'alpha granularity' in the market is critical. By this, we mean the concentration of alpha opportunities across top-down 
and bottom-up decisions. Cross-sectional return dispersion and its sources are important metrics for alpha granularity. Our modelling of return dispersion uncovers several attractive characteristics - we show how it can be decomposed into separate topdown and bottom-up components, in line with an active investment process. Such delineation can provide guidelines for designing portfolio bet structures.

We also show the exact mathematical linkage between (cross-sectional) return dispersion and (time-series) volatility and correlation, and focus on the impact of changes in volatility and correlation on available alpha opportunities. We highlight possible hedging strategies to protect against changes in return dispersion.

\section{Previous studies}

There have been studies on the broad subject of 'alpha granularity' by examining return dispersion, which is closely related to the notion of diversification from a risk management perspective. Solnik and Roulet (2000) introduced a metric of cross-sectional correlation to estimate global correlation levels and trends of equity returns via a single-factor model. It is highlighted that maximising global dispersion, thus minimising global correlation, should be a major objective of global asset allocation. This aim should be an evolving target contingent on the prevailing correlation structure of global markets. To this end, a tractable measure of 'alpha availability' should be useful to gauge the potential success of differentiating winners from losers. Hwang and Salmon (2004) developed statistical measures to test for herding or contagious behaviours in different stock markets.

Intrinsic to the application of crosssectional volatility in any investment process is its close relationship with time-series volatility - linkages between these two volatility variables and their statistical properties were examined in great detail in
Hwang and Satchell (2001) via linear factor models. There have also been attempts to incorporate the former in forecasting both market risk and market returns. See, for example, Hwang and Satchell (2005) and Goyal and Santa-Clara (2003), respectively for these two research areas.

While previous studies have largely focussed on unravelling the mathematical properties of return dispersion, which provides an extra dimension of understanding in stock co-movements within a given markets, less emphasis has been placed on how to incorporate this additional information in the area of portfolio construction. In this paper, we propose a new framework to study crosssection volatility in a portfolio context and its relation to overall market return distribution. It is our contention that establishing such a linkage is useful in channelling optimal research resources under constraints for an active asset manager in the reality world.

This paper is organised as follows: in the next section, we revisit the use of linear factor models to ascertain any herding effects in a given market. In the following section, we introduce our decomposition approach in separating 'alpha budget' into top-down asset allocation and bottom-up stock selection components. In the penultimate section, we establish a linkage between cross-sectional dispersion and timeseries correlation and volatility using continuous-time financial mathematics. Conclusions follow in the last section.

\section{Return dispersion and alpha opportunity}

The evolution of cross-sectional return dispersion over time - the distribution of asset returns - is an important metric in determining both the absolute and relative attractiveness of investment strategies. Crosssectional dispersion can be described by a number of different metrics, such as range, inter-quartile range and mean absolute deviation. Cross-sectional volatility is a 
particularly attractive metric for dispersion, since it considers the entire collection of returns from a sample at a given point in time. In its unweighted form, cross-sectional variance (or volatility squared) is given by:

$$
\chi_{t}^{2}\left(r_{t}\right)=\frac{1}{N} \sum_{i=1}^{N}\left(r_{i t}-r_{m t}\right)^{2}
$$
where $r_{m t}=1 / N \sum_{i=1}^{N} r_{i t}$ is the unweighted ${ }^{1}$
market return.

Alternatively, in matrix form $\chi_{t}^{2}=\mathbf{r}_{t}^{\mathrm{T}} \mathbf{M} \mathbf{r}_{t}$ where $\mathbf{r}_{t}^{\mathrm{T}}=\left(r_{1}, r_{2 t}, \ldots, r_{N t}\right)$, that is, the vector of asset returns and $\mathbf{M}=1 / \mathrm{N}\left(\mathbf{I}-\left(\mathbf{1} \times \mathbf{1}^{\mathrm{T}} / N\right)\right)$ with a conformable identity matrix and two conformable vectors of ones.
Throughout, we use dispersion and crosssectional standard deviation of returns $\left(\chi_{t}\right)$ interchangeably.

Figure 1 shows the evolution of this crosssectional dispersion measure for MSCI Europe and MSCI US, together with their (time-series) market volatility and cumulative (equally-weighted) market returns. On the face of it, they appear to confirm the view that the current market is characterised by low risk and low (excess) stock alpha. It appears that the market factor is at work in pinning down dispersion - most of the absolute return opportunities come from market effects. This raises the question of where the remaining alpha opportunities
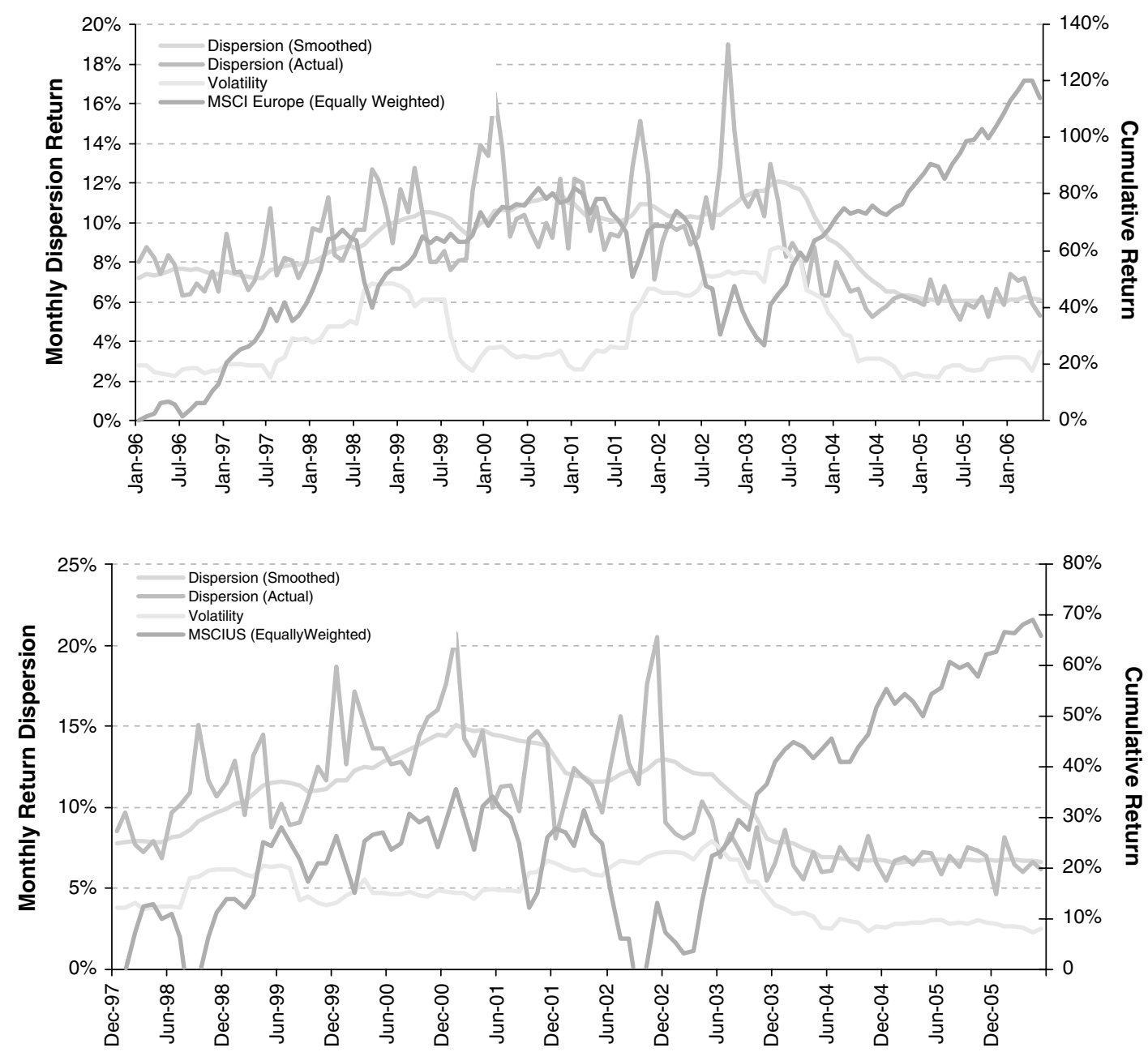

Figure 1 Comparison of dispersion with volatility 
reside, which should guide both research efforts and risk allocation decisions.

\section{Herding effect - Market beta compression}

We can utilise our dispersion metric to investigate stock return homogeneity under varying market regimes - changes in dispersion can be linked to broad market factors. This may be particularly relevant for long-only (long-short) investors with specific (net) beta targets. Low beta dispersion could lead to unintended market neutrality in portfolios. Particularly for active managers, this might mean that little incremental alpha is being generated from stock selection, with the fund only being compensated for market factor risk.

Active managers may want to avoid stocks that show a 'herding' tendency towards the market.

Previous research such as Hwang and Salmon (2004) has studied market herding behaviours in a tractable framework. The objective of this subsection is to re-visit the problem with emphasis on empirical results.

We now use a single-index market model to study individual stock returns (assuming zero risk-free interest rate):

$$
r_{i t}=\beta_{i t} r_{m t}+\varepsilon_{i t}
$$

where $r_{m t}$ is the unweighted market return defined above.

Plugging this model into our first equation, we can easily extract the beta dispersion component from total return dispersion, as follows $\left(\right.$ let $\chi_{t}^{2}(\cdot)$ denote the dispersion operator at time $t$ ):

$$
\begin{aligned}
\chi_{t}^{2}\left(r_{t}\right) & =\frac{1}{N} \sum_{i=1}^{N}\left(r_{i t}-r_{m t}\right)^{2} \\
& =\frac{1}{N} \sum_{i=1}^{N}\left(\left(\beta_{i t}-1\right) r_{m t}+\varepsilon_{i t}\right)^{2} \\
& \approx \frac{1}{N} \sum_{i=1}^{N} r_{m t}^{2} \cdot\left(\beta_{i t}-1\right)^{2}+\frac{1}{N} \sum_{i=1}^{N} \varepsilon_{i t}^{2} \\
& =r_{m t}^{2} \cdot \chi_{t}^{2}\left(\beta_{t}\right)+\chi_{t}^{2}\left(\varepsilon_{t}\right)
\end{aligned}
$$

Here it is assumed that systematic and idiosyncratic risks in this model are also cross-sectionally uncorrelated, and residuals terms have zero cross-sectional expectation.

Hence, under the assumption that the residuals $\varepsilon_{i t}$ are uncorrelated with the market return $r_{m t}$ and to each other, it is shown that the total stock dispersion metric can be expressed as a function of beta dispersion and non-market return dispersion.

Figure 2 shows the evolution of beta dispersion for Europe and the US. Both markets show beta compression, with beta dispersion dropping from highs of around 1.4 in 2001 to lows of around 0.7 in 2003.

Herding towards the market portfolio started earlier in Europe than in the US. Beta dispersion in the US has recovered somewhat since the lows in 2003, while that in Europe has continued to stay low. At the same time, overall dispersion in both markets has remained low — reflecting low market volatility.

We want to investigate any size effects despite time-varying dispersion. It is trivial to sort companies by market capitalisation, bucket them into deciles and compare their relative strengths of contributions (see Figure 3). We note the following in the European context:

- Large Caps (Decile 10) show significant herding towards the market in Europe in aggregate, mega-cap returns form a proxy for the market, and are driven by big-picture macro-economic and sector effects.

- Small Caps (Decile 1) show less herding behaviour and seem driven more by idiosyncratic effects, such as corporate restructuring, earnings surprises and product innovations. These companyspecific risks are typically uncorrelated with the market and may reflect lower analyst coverage and liquidity. We also found considerable variability among Mid Caps (average of Deciles 4-6). 

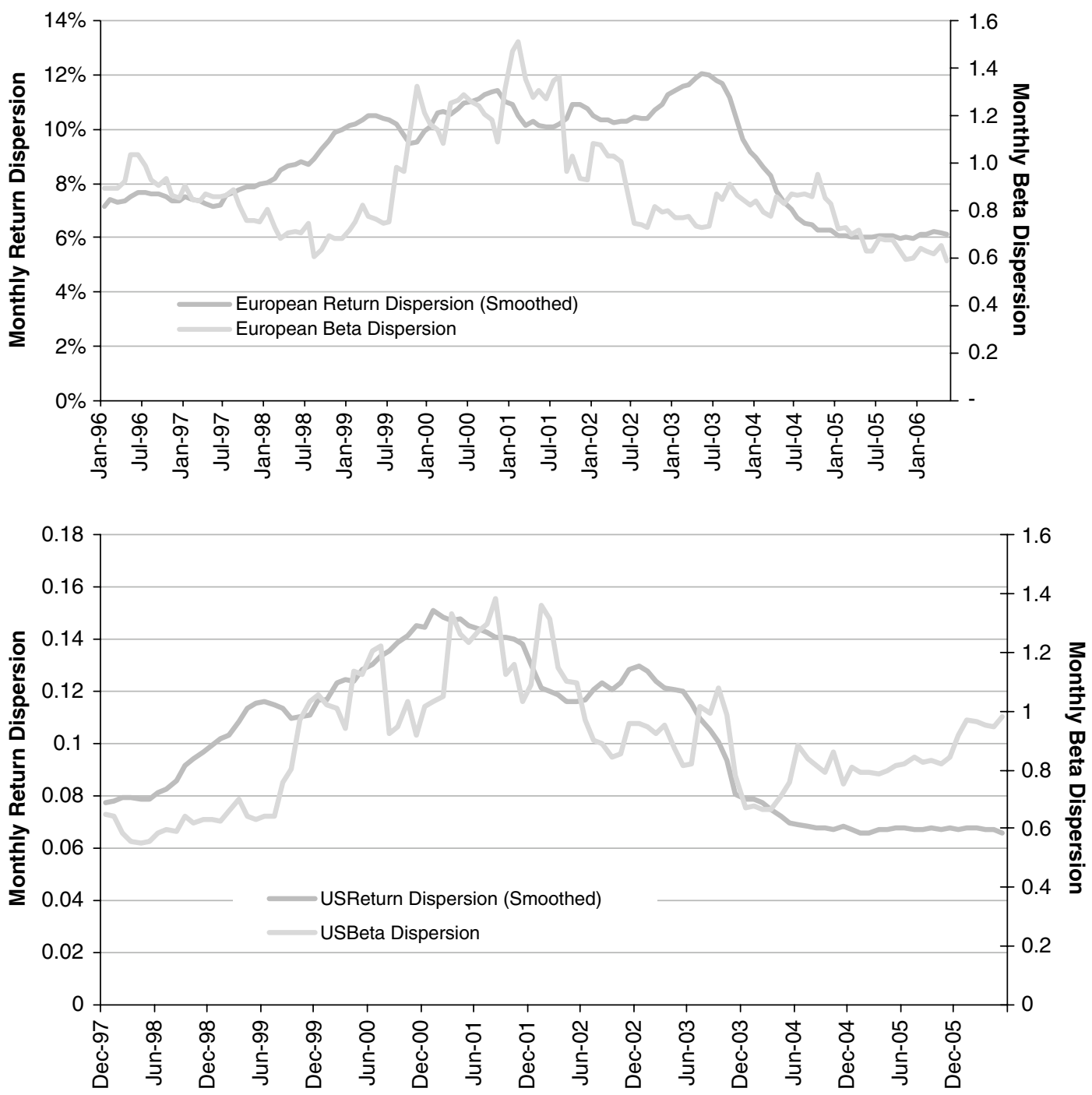

Figure 2 Market beta dispersion

Our use of an equal-weighted benchmark should control for any size bias inherent in a 'standard' (market-cap weighted) benchmark.

\section{Non-market return compression}

Active managers typically focus on the nonmarket component of stock returns and seek stock-specific alpha opportunities. The decomposition of total return into market and idiosyncratic components allows us to investigate the evolution of the alpha hunting ground for those investors as well. In this section, we focus on the dispersion of nonmarket returns, $\chi_{t}^{2}\left(\varepsilon_{t}\right)$. This metric is particularly relevant for market-neutral strategies.

Figure 4 shows the evolution of nonmarket return dispersion compared to total return dispersion for both Europe and the US. Non-market return dispersion compressed significantly during the market rebound in 2003 and has not recovered since. Combined with the current beta compression, this has led to shrinking of residual return opportunities, particularly 

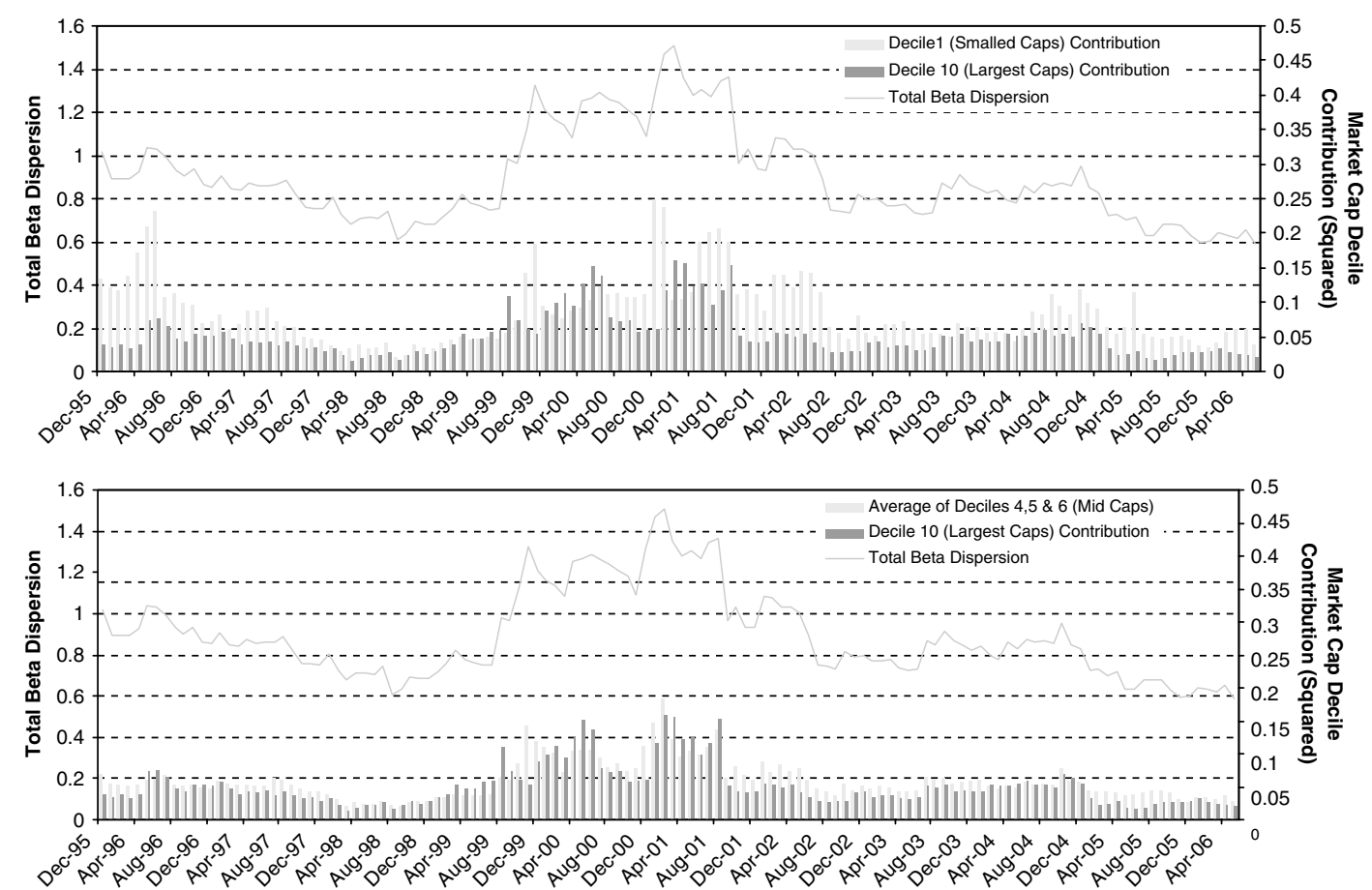

Figure 3 Contributions to market beta dispersion-size analysis

during the second part of 2005. Lower than average performance of some hedge funds during that period may well be attributable to this compression. Overall, non-market return dispersion is highly correlated with the total return dispersion. Studying the size and the sources of non-market return dispersion can be helpful in determining the optimal investment approach at any one point in time.

In the European market, the relative importance of market beta and non-market stock dispersion went through several characteristic episodes during our sample period, which can serve as examples:

- Relatively high non-market dispersion with market beta compression (July to December 1998, July 2004 to July 2005). These periods of decoupling between non-market dispersion and market beta dispersion promise the relatively greater rewards from active stock selection, particularly in a market-neutral setting. Note that the two periods differ in absolute return opportunities - the latter period's opportunity set was much smaller.

- High non-market return dispersion with high market beta dispersion (1999-2002).

These periods of coupling between market and non-market risks require a careful resource allocation between macro (eg tactical asset allocation) and micro (eg fundamental research allocation) stock return drivers. The period encompasses both the latter part of the TMT bull market, where sector effects were important sources of returns, and the bear market following, where optimal market exposure and stock selection were critical.

- Low absolute non-market dispersion with market beta compression (from 2004 onwards). Strong market herding and a lack of residual return opportunities make generating returns from active stock selection challenging, particularly for betaneutral funds. 

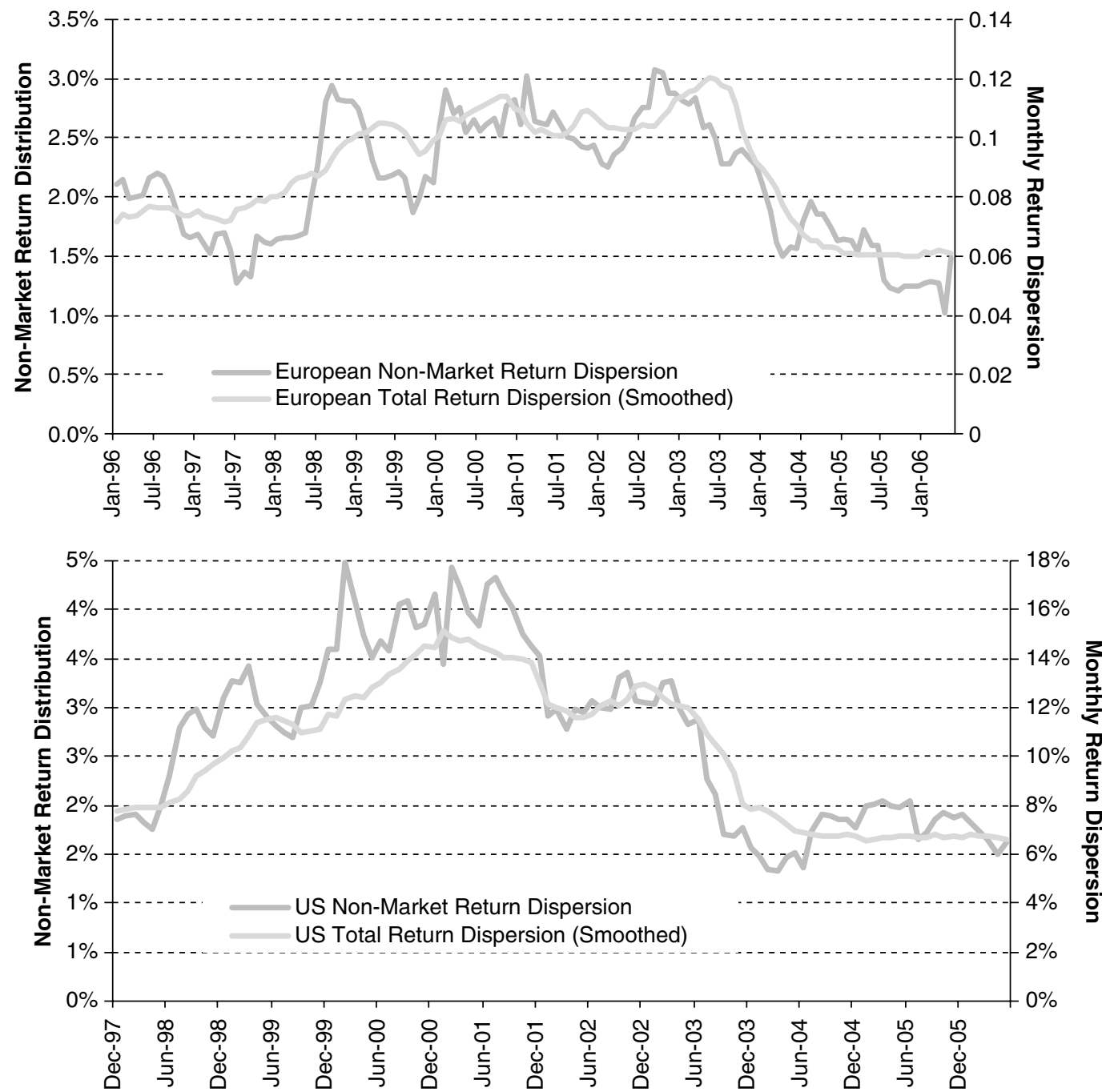

Figure 4 Non-market return dispersion

\section{Concept of alpha granularity}

A measure of alpha granularity, or the 'availability' of disparate alpha opportunities, should shed light on the optimal concentration of stocks in the portfolio. Such a measure, however, is often difficult to gauge quantitatively.

Cross-sectional dispersion is closely related to this concept of alpha granularity what proportion of the return potential is generated from a given coverage level. In fact, we can treat dispersion as a hypothetical perfect-foresight scenario, which can serve as an objective benchmark for long/short fund managers.

To see this interpretation, we devise a perfect-foresight portfolio policy as follows:

Let

$$
w_{i t}=r_{i t}-r_{m t},
$$

where

$$
r_{m t}=1 / N \sum_{i=1}^{N} r_{i t}
$$

is the unweighted market return. 
Written this way, $w_{i t}$ is the amount of money invested in stock $i$ (ignoring the notional amount of proportionality here). Under this assumption, the long or short exposure of each security is directly linked to the subsequent return; as such, the fund size is variable in time according to alpha availability.

Trivially, $\sum_{i=1}^{N} w_{i t}=0$, namely this is a dollar-neutral 'hedge fund'.

As a result, the portfolio profit over a single period $t$ can be written as:

$$
\begin{aligned}
& \sum_{i=1}^{N} w_{i t} r_{i t} \\
&=\sum_{i=1}^{N}\left(r_{i t}-r_{m t}\right) r_{i t} \\
&=\sum_{i=1}^{N}\left(r_{i t}-r_{m t}\right) \cdot r_{i t}-\sum_{i=1}^{N}\left(r_{i t}-r_{m t}\right) \cdot r_{m t} \\
&\left(\sum_{i=1}^{N}\left(r_{i t}-r_{m t}\right) \cdot r_{m t}=0\right) \\
&= \sum_{i=1}^{N}\left(r_{i t}-r_{m t}\right)^{2} \\
&= N \cdot \chi_{t}^{2}
\end{aligned}
$$

(ie $N$ times the equally-weighed dispersion).

Hence, the portfolio return is identical to cross-sectional dispersion up to a proportional constant.

\section{Alpha budgeting - Country, sector, size, style and beyond}

Our dispersion-based analysis of the turbulent equity market environment, ranging from extreme market oscillations to the current relatively low volatility environment, shows that maintaining discipline and a realistic assessment of the risk and return potential is critical. We now use dispersion as our diagnostic tool to investigate the relative importance of different top-down asset classes and the impact this may have on the organisation of an active investment process.

We first revisit the country versus sector debate for European markets. ${ }^{2}$ Figure 5 shows the average top-down dispersion across countries and industry groups, as well as the average correlation since 1995.

Our results reaffirm our previous conclusions - in Europe, since 1998, industry group return dispersion has been consistently higher than that of countries, even though both have declined since 2002 . Average correlation has been lower across industry groups than countries since 1998 . Return opportunities from industry group allocation are therefore relatively greater than those from country allocation - investors should continue to adopt a sector approach to top-down allocation in Europe. ${ }^{3}$

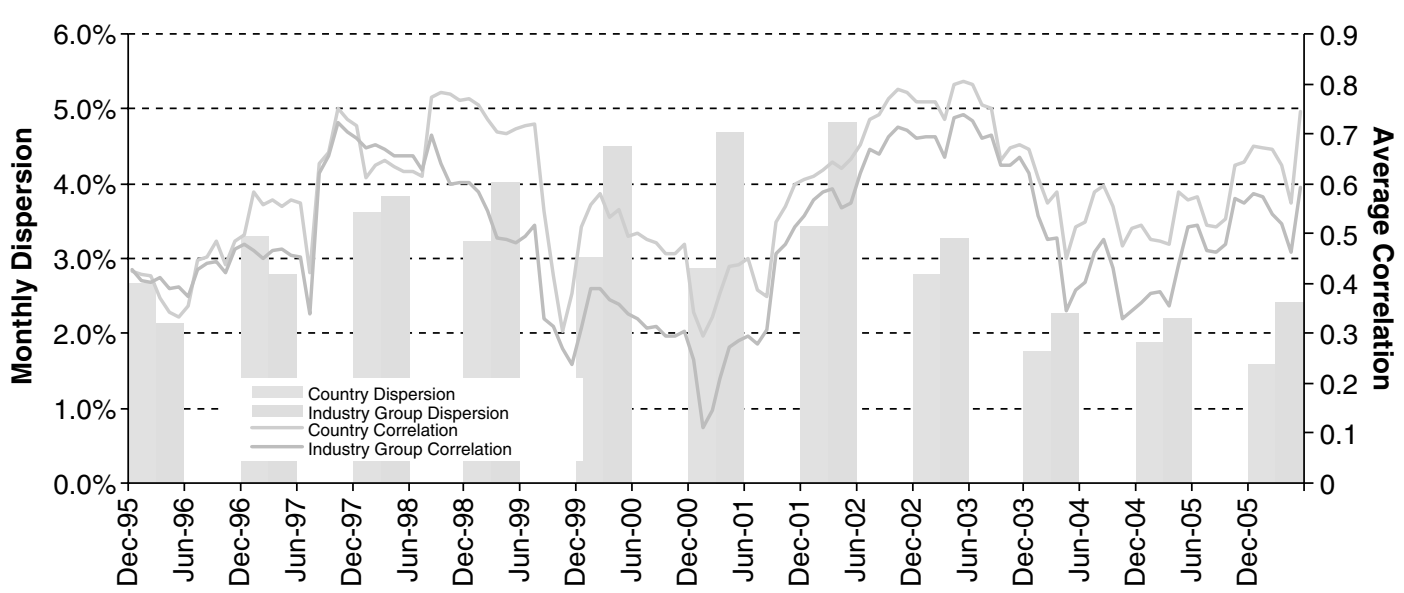

Figure 5 Country vs. industry group rotation in MSCI Europe 
We can link the top-down dispersion with our earlier total return dispersion metric. This will enable us to introduce the concept of alpha budgeting. We decompose total cross-sectional dispersion in the market into two 'independent' components, top-down and bottom-up contribution:

$$
\begin{aligned}
\chi_{t}^{2}= & \chi_{\text {Top-Down Asset Allocation, } t}^{2} \\
& +\chi_{\text {Average Bottom-Up Stcok Selection, } t}^{2}
\end{aligned}
$$

This clean, orthogonal relationship (see Figure 6) can provide a standardised comparison of different fund organisation schemes and is derived as follows:

Denote, at time $t$, the following:

$I_{j}$ : number of stocks belonging to group $j$ $w_{i j t}$ : weight of stock $i$ belonging to group $j$ $r_{i j t}$ : return of stock $i$ belonging to group $j$

We also define the following

$r_{j t}$ : weighted return of group $j$.

$$
r_{j t}=\sum_{i=1}^{I_{j}} \frac{w_{i j t} \cdot r_{i j t}}{w_{j t}}
$$

where $w_{j t}=\sum_{i=1}^{I_{j}} w_{i j t}$ is the weight of group $j$ in the market.

$r_{t}$ : weighted return of the market.

$$
r_{t}=\sum_{j=1}^{J} \sum_{i=1}^{I_{j}} w_{i j t} \cdot r_{i j t}
$$

$$
\begin{aligned}
\chi_{t}^{2}= & \sum_{j=1}^{J} \sum_{i=1}^{I_{j}} w_{i j t}\left(r_{i j t}-r_{t}\right)^{2} \\
= & \sum_{j=1}^{J} \sum_{i=1}^{I_{j}} w_{i j t}\left(r_{i j t}-r_{j t}+r_{j t}-r_{t}\right)^{2} \\
= & \sum_{j=1}^{J} \sum_{i=1}^{I_{j}} w_{i j t}\left(r_{j t}-r_{t}\right)^{2}+\sum_{j=1}^{J} \sum_{i=1}^{I_{j}} w_{i j t}\left(r_{i j t}-r_{j t}\right)^{2} \\
& +2 \cdot \sum_{j=1}^{J} \sum_{i=1}^{I_{j}} w_{j t}\left(r_{j t}-r_{t}\right)\left(r_{i j t}-r_{j t}\right) \\
= & \sum_{j=1}^{J} w_{j t}\left(r_{j t}-r_{t}\right)^{2}+\sum_{j=1}^{J} w_{i j t} \cdot \chi_{\text {stock within group } j}^{2} \\
& +2 \cdot \sum_{j=1}^{J}\left(r_{j t}-r_{t}\right) \sum_{i=1}^{I_{j}} w_{i j t}\left(r_{i j t}-r_{j t}\right) \\
= & \left.\chi_{\text {top-down }, t}^{2}+\frac{\chi_{\text {bottom-up }, t}^{2}}{I_{j}} w_{i j t}\left(r_{i j t}-r_{j t}\right)=0 \quad \forall j\right) \\
& \quad\left(\sum_{i=1}\right)
\end{aligned}
$$

(Setting $w_{i j t}=1 / N$ gives the unweighted results, where the total number of stocks, $N=\sum_{j=1}^{J} I_{j}$ ).

This decomposition of dispersion allows us to compare, on a common basis, the relative alpha opportunity set of classifying stocks along country, sector, size or style lines. ${ }^{4}$ Figure 7 shows the relative strengths of different organisation methods under different market regimes — the 'alpha pies'. The diameter of each pie represents the total return dispersion - the opportunity space for alpha budgeting. The 'short' leg of each right-angled triangle then shows the topdown contribution from a given asset class

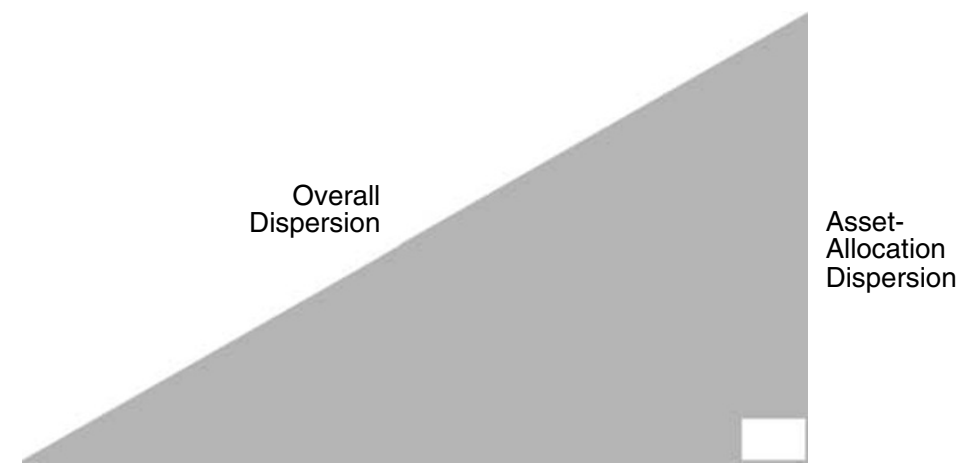

Average Stock-Selection Dispersion

Figure 6 Dispersion decomposition-top-down/bottom-up 


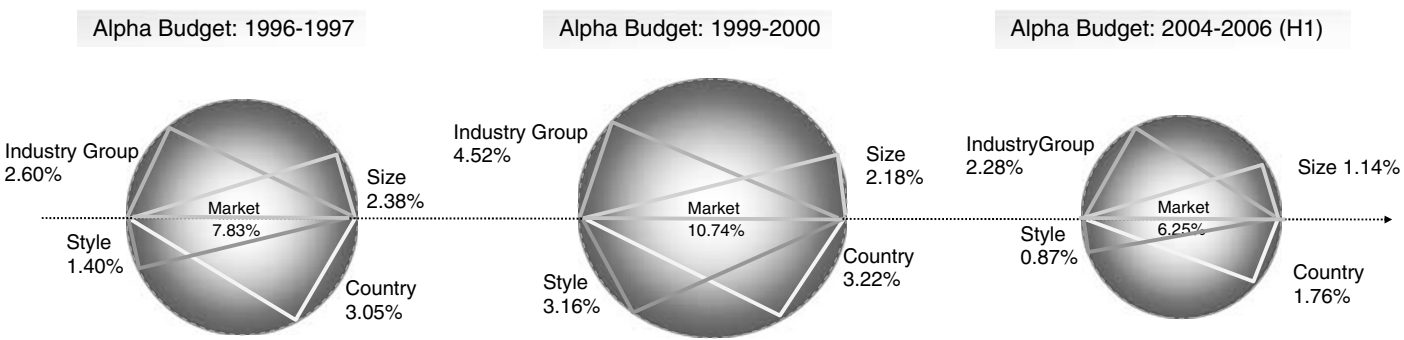

Figure 7 Alpha budgeting in $\mathrm{MSCl}$ Europe (monthly returns)

definition, and the longer leg represents the remaining stock level contribution. We can draw some tentative conclusions from the alpha budgets in MSCI Europe:

- Sector rotation wins over country allocation - compared to the 1996-97 period, industry group top-down dispersion is greater, in absolute terms, than country dispersion for subsequent periods.

- Size matters - segregation of the market into market cap buckets can lead to significant top-down dispersion opportunities. Systematic granular (eg decile-based) size bets are, however, not achievable in practice, and we view coarse (large versus small cap) size bets as an important decision at least from a risk control viewpoint.

Despite the low total return dispersion pattern in global markets at the moment, the comparison between alpha opportunities from different top-down classifications shows that it remains critical to choose the appropriate approach to top-down fund organisation and research allocation.

This decomposition approach can be easily applied on a portfolio level to study the active 'bet structure' relative to the dispersion patterns in the market. We link the relative bet structure to the active skills of the portfolio manager, and argue that our dispersion analysis can add insight into the optimal dispersion distribution across the portfolio. By doing so, we can show the relative aggressiveness in top-down and bottom-up alpha budgeting in a given portfolio compared with the current market alpha budget. As noted earlier, any definition of top-down asset class can be used. Our approach therefore allows a portfolio manager to investigate the alpha contribution of both explicit (eg sector or style) and implicit (eg size or beta) bets.

Table 1 compares a European Model Portfolio ${ }^{5}$ with the MSCI Europe benchmark to show our approach. We track the portfolio exposure to sectors as top-down asset classes.

Taking sectors as the top-down asset class, the ratio of bottom-up stock dispersion (standard deviation) to top-down dispersion is around 4.5:1 in MSCI Europe, and around 1.8:1 for the European Model Portfolio. The portfolio concentrates its bets relatively more on variability in sectors rather than on stocks. In general, any deviation between portfolio and benchmark should be driven by forwardlooking alpha expectations, as well as relative skill sets.

Overall, the portfolio's total return dispersion is around 1.32 per cent, against 1.54 per cent for the benchmark - this is not surprising, given the number of names in this concentrated portfolio relative to the benchmark. Given the larger sector return dispersion (0.64 per cent for the portfolio versus 0.33 per cent for the benchmark), the bottom-up stock bet dispersion within sectors is smaller in the portfolio than in the benchmark. Table 1 shows that this holds for most sectors - however, contrast the 
Table 1 Dispersion-based portfolio bet structure (daily returns)

\begin{tabular}{|c|c|c|c|c|}
\hline & European model portfolio & & MSCI Europe & \\
\hline Total dispersion ${ }^{\mathrm{a}}$ & 175.0 & & 238.5 & \\
\hline Top-down sector allocation & 41.2 & & 11.2 & \\
\hline Bottom-up stock selection & 133.8 & & 227.3 & \\
\hline Consumer discretionary & & 33.5 & & 40.8 \\
\hline Consumer staples & & 6.4 & & 12.2 \\
\hline Energy & & 5.1 & & 7.7 \\
\hline Financials & & 21.5 & & 39.9 \\
\hline Healthcare & & 7.0 & & 24.2 \\
\hline Industrials & & 4.5 & & 43.5 \\
\hline Information technology & & 11.1 & & 19.3 \\
\hline Materials & & 29.8 & & 26.6 \\
\hline Telecom services & & 11.1 & & 7.6 \\
\hline Utilities & & 3.7 & & 5.5 \\
\hline
\end{tabular}

${ }^{a}$ Notes: All figures are quoted in variance terms and multiplied by $10^{\wedge} 6$ for better clarity.

aggressive bottom-up dispersion in the Materials sector to the minimal dispersion in the Industrials sector. Since the portfolio is highly concentrated in its name coverage, some degree of active bottom-up alpha bets is expected. The portfolio is relatively active in stock selection within the Telecom Services and Consumer Discretionary sectors.

The ordering of bottom-up stock dispersion in the portfolio does not necessarily need to follow that of the benchmark - just as for the top-down/ bottom-up dispersion decomposition, bottom-up stock dispersion decisions should correspond to alpha expectations and stock picking skills within sectors. The rank correlation between the bottom-up dispersion in this portfolio and the benchmark is 0.42 - this simple metric is an indication that, in this case, the stock selection budgets are not too far away from the alpha availability within the market.

In general, discrepancies between bottom-up dispersion in portfolio and benchmark should be due to strategic active alpha bets, or higher alpha convictions in some sectors. Our analysis can spotlight potentially unintended discrepancies, such as an emphasis on sectors with relatively high homogeneity, or a size bet structure that is not aligned with targeted alpha budgets.
These might be indications of sub-optimal portfolio policies.

\section{Multi-staged portfolio design}

This decomposition is flexible enough to be adapted to any top-down classification schemas provided they are exhaustive and exclusive. We can also extend the decomposition technique to multiple topdown classification layers, for example, a sector allocation - size sub-allocation stock selection partitioning. ${ }^{6}$ Figure 8 shows the modified alpha-budget sets schematically.

The dispersion ratio (relative size of topdown versus bottom-up dispersion) has implications for any investment process involving multi-stage portfolio construction or multiple alpha sources. Quite often, topdown and bottom-up portfolio construction is managed separately in typical asset management organisations, or top-down allocation may be a by-product of the bottom-up process.

The above technique allows us to define a dynamic weighting between the decisionmaking levels, driven by the prevailing dispersion ratio (the ratio of the two shorter sides of the right-angled triangle). If we have top-down and bottom-up processes each giving target stock weights, we can use the dispersion ratio to determine the overall active stock weight of the combined two- 

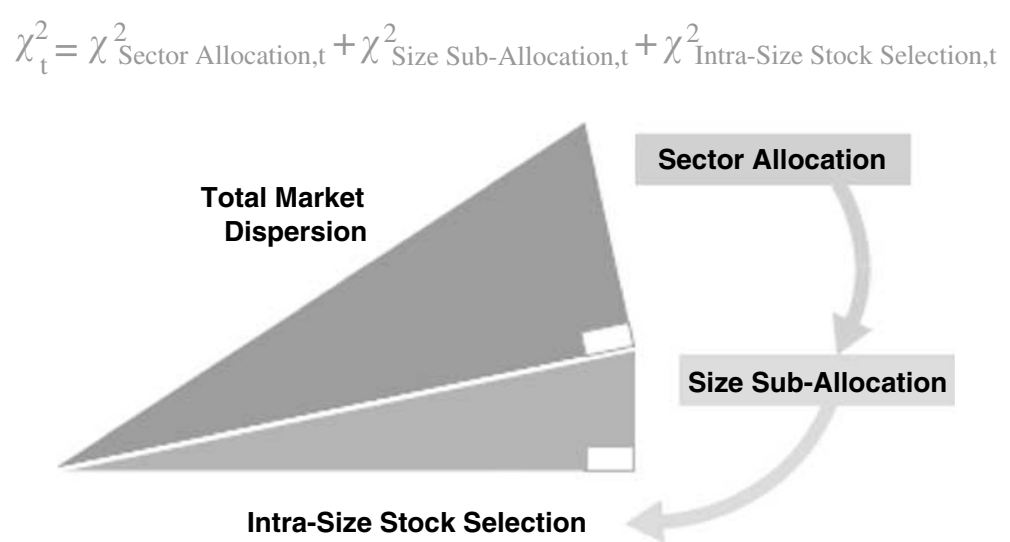

Figure 8 Multi-staged alpha-budgeting

stage model by

$$
\begin{aligned}
w_{i t} & =\Omega_{1} \cdot w_{i}^{\text {Sector Allocation }, t} \\
& +\Omega_{2} \cdot w_{i}^{\text {Stock Selection }, t}
\end{aligned}
$$

where $\Omega_{1}$ and $\Omega_{2}$ are risk allocation factors that are set dynamically to reflect the dispersion ratio as well as the alpha quality of each decision level. This exercise can therefore assist in targeting risk budgets in the investment process.

\section{Linking cross-sectional and time- series volatilities}

Knowledge of total return distribution and its decomposition into coverage buckets, as well as top-down and bottom-up contribution to dispersion, can be very useful diagnostics to develop 'optimal' alpha budgeting. A logical question to ask is whether we can model future or predicted dispersion. This is no less challenging than forecasting volatility; however, we show that there is an analytical linkage between timeseries volatility and cross-sectional dispersion.

This relation can be established algebraically, and we show later that expected cross-sectional dispersion can be given by the following:

$$
\begin{aligned}
E_{t}\left[\chi_{t}^{2}\right]= & \left(\sum_{i=1}^{N} w_{i t} \sigma_{i t}^{2}-\sigma_{m t}^{2}\right) \\
& -\sum_{i=1}^{N}\left(w_{i t} \mu_{i t}-\mu_{m t}\right)^{2}
\end{aligned}
$$

We establish that expected dispersion can be decomposed into two terms: (1) differential between average stock volatility and market volatility and (2) dispersion among expected stock returns. (Note that this generic linkage does not depend on specification of the underlying returngenerating process.)

Proof: If we replace $r_{i t}$ by $R_{i t}$ to denote the random variable corresponding to a returngenerating process, we can express the expectation of cross-sectional volatility in time, as follows.

$$
\begin{aligned}
& E_{t}\left[\chi_{t}^{2}\left(R_{t}\right)\right]=E_{t}\left[\sum_{i=1}^{N} w_{i t}\left(R_{i t}-R_{m t}\right)^{2}\right] \\
& \text { where } R_{m t}=\sum_{i=1}^{N} w_{i t} R_{i t} \text { is the weighted market return } \\
& =E_{t}\left[\sum_{i=1}^{N} w_{i t} R_{i t}^{2}-2 \cdot R_{m t} \sum_{i=1}^{N} w_{i t} R_{i t}+R_{m t}^{2} \cdot \sum_{i=1}^{N} w_{i t}\right] \\
& =E_{t}\left[\sum_{i=1}^{N} w_{i t} R_{i t}^{2}-R_{m t}^{2}\right] \\
& =\sum_{i=1}^{N} w_{i t} \cdot E_{t}\left[R_{i t}^{2}\right]-E_{t}\left[R_{m t}^{2}\right] \\
& =\sum_{i=1}^{N} w_{i t}\left(\sigma_{i t}^{2}+\mu_{i t}^{2}\right)-\left(\sigma_{m t}^{2}+\mu_{m t}^{2}\right) \\
& =\left(\sum_{i=1}^{N} w_{i t} \sigma_{i t}^{2}-\sigma_{m t}^{2}\right)+\sum_{i=1}^{N} w_{i t}\left(\mu_{i t}-\mu_{m t}\right)^{2}
\end{aligned}
$$

(Setting $w_{i t}=1 / N$ gives the unweighted results). 


\section{Understanding correlation (and when it hurts)}

To appreciate how short-term fluctuations in dispersion are influenced by individual stock return variations, one can ignore the second term in the last expression. To see why this is possible, we look at the continuoustime case. Assume the log-prices, $X_{i t}$ 's, of assets in a stock universe, take the following simple multivariate diffusion specification at time $t$ :

$$
\mathrm{d} X_{i t}=\mu_{i} \mathrm{~d} t+\sigma_{i} \mathrm{~d} B_{i}
$$

where $B_{i}$ 's are Brownian motions with $\mathrm{E}\left\lfloor\mathrm{d} B_{i}, \mathrm{~d} B_{j}\right\rfloor=\rho_{i j} \mathrm{~d} t$.

When the above return-generating processes are used as inputs $\mathbf{r}^{\mathrm{T}}=\left(\mathrm{d} X_{1 t}, \mathrm{~d} X_{2 t}, \ldots, \mathrm{d} X_{N t}\right)$ in our expectation operator shown before, one obtains the following:

$$
\chi_{(t, t+\mathrm{d} t)}^{2}=\left(\sum_{i=1}^{N} w_{i t} \sigma_{i t}^{2}-\sigma_{m t}^{2}\right) \mathrm{d} t
$$

The second term involving expected returns disappears owing to the fact that terms containing $\mathrm{d} t$ of order higher than unity vanish in the limiting process. By the same token, since the second-moment expectation and thus the variance also yield to nullity, and that is why we drop the expectation sign here.

To summarise, over a short period, dispersion changes are seen to be driven by individual volatility and the overall market volatility only.

With this in mind, we can use some approximation techniques to show the interplay between market and stock volatilities, as well as their correlations. For example, one can introduce the following generalisations in the unweighted case:

Short-term dispersion (dropping time subscripts for simplicity here):

$$
\begin{aligned}
& \propto \frac{1}{N} \sum_{i=1}^{N} \sigma_{i}^{2}-\sigma_{m}^{2} \\
& =\frac{1}{N} \sum_{i=1}^{N} \sigma_{i}^{2}-\frac{1}{N^{2}} \sum_{i=1}^{N} \sigma_{i}^{2}-\frac{1}{N^{2}} \sum_{j \neq k}^{N} \sigma_{j k}^{2} \\
& =\frac{N-1}{N^{2}} N \overline{\sigma_{i}^{2}}-\frac{1}{N^{2}} N(N-1) \overline{\sigma_{j k}^{2}} \\
& \approx \overline{\sigma_{i}^{2}}-\overline{\sigma_{j k}^{2}}
\end{aligned}
$$

(when $N$ is sufficiently large and $j \neq k$ ).

The last expression is simply the differential between the average stock

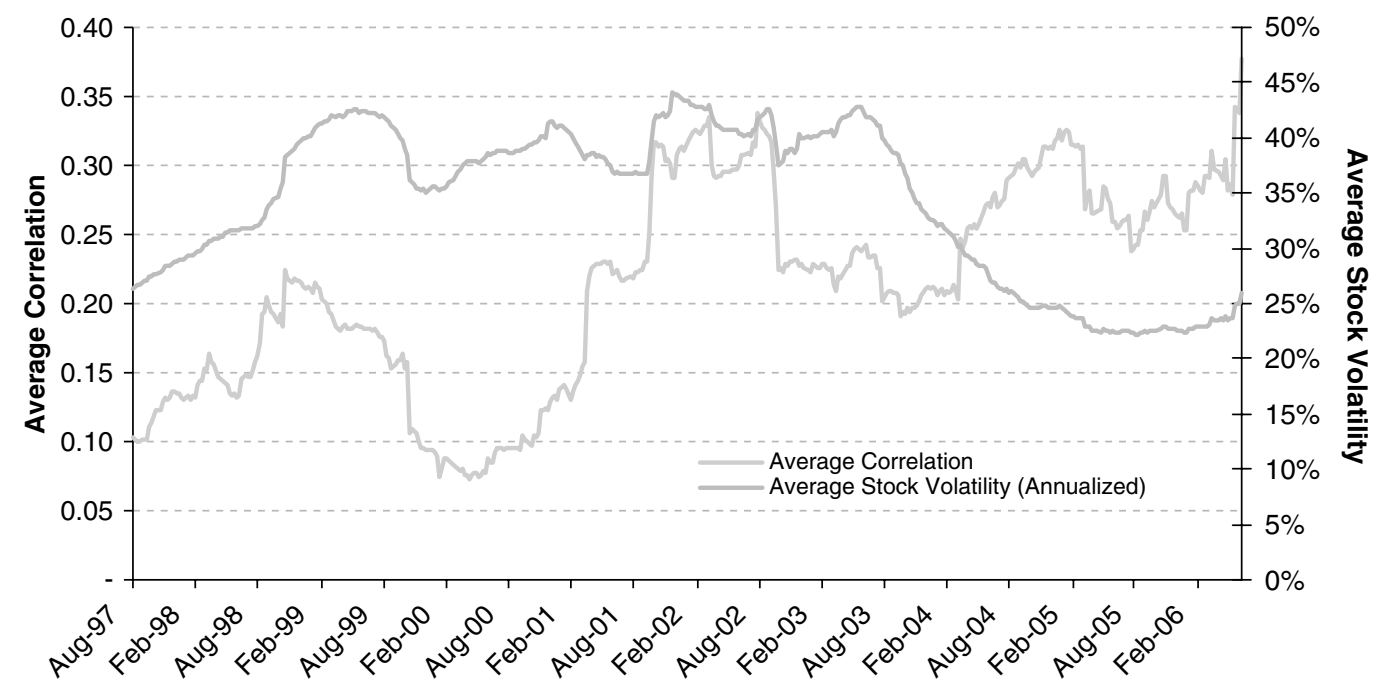

Figure 9 Drivers of dispersion in $\mathrm{MSCl}$ Europe: Average stock volatility and correlation (using weekly returns) 
variance and the average covariance between any two stocks.

This delineation allows us to appreciate why dispersion falls when overall market volatility declines, since the first term (which measures the overall riskiness of composite assets) moves linearly with market dispersion. The second term, which measures the comovements of assets, reduces dispersion when being positive and vice versa.

Alternatively, one can rewrite our approximation further as $\overline{\sigma_{i}^{2}}(1-\bar{\rho})$ to clearly see the negative contribution to dispersion by correlation risk where $\bar{\rho}=\overline{\sigma_{j k}^{2}} / \overline{\sigma_{i}^{2}}$ for $j \neq k$. Dispersion is therefore expected to fall when average stock volatility falls and/or when average correlation rises.

Shrinking dispersion is one of the major risks confronting long/short investments. Since dispersion is positively related to volatility, and negatively to correlation, a possible partial hedge against declining dispersion can be achieved through a short volatility and long correlation position. In terms of financial instruments, variance swaps and forward-starting variance swaps, and dispersion trades can be used as synthetic tools to gain such exposures.

As a final illustration, Figure 9 shows the contribution of average stock volatility and correlation to the overall MSCI Europe return dispersion. The current market environment is clearly a 'double whammy' for investors - low stock volatility, plus high and rising average correlation.

\section{Conclusions}

Cross-sectional return dispersion can be an attractive metric for alpha granularity in markets. Our overview of dispersion patterns in regional markets and a decomposition approach into top-down and bottom-up contribution to dispersion highlights several features of the current market environment. For long/short investors in particular, our dispersion decomposition can help optimise investment strategy over time, with practical insights into the 'optimal' matching of research coverage with alpha opportunities.

We showed how this dispersion decomposition can be used in practical alpha budgeting for portfolio construction and monitoring. Our diagnostic tools add an extra level of understanding to portfolio analysis, and can help to identify any unintended discrepancies between portfolio bet structure and market alpha budgets.

We also establish a linkage between crosssectional return dispersion and (time-series) volatility. We develop the analytical linkage between the two, and show that dispersion can be seen as a function of average stock variance and average correlation between stocks. This contributes to a better understanding of return dispersion. We highlight hedging possibilities in practice involving variance swaps and dispersion trades, which can limit the exposure to (shrinking) return dispersion.

Our new analytical framework makes a meaningful contribution to risk management in general and to the study of alpha granularity in particular. Quantitative analysis of alpha granularity can yield insights into optimal portfolio construction, research coverage and organisation. It is expected that research in this area would advance in the future.

\section{Acknowledgments}

The authors thank Steve Satchell of Trinity College, Cambridge, for valuable discussions.

\section{Notes}

1. Dispersion is most commonly measured using unweighted returns. A minor modification can give the market cap weighted version: $\chi_{t}^{2}\left(r_{t}\right)=\sum_{i=1}^{N} w_{\text {it }}\left(\mathrm{r}_{\mathrm{it}}-\mathrm{r}_{\mathrm{mt}}\right)^{2}$, where $\mathrm{r}_{\mathrm{mt}}=\sum_{i=1}^{N} w_{i t} r_{i t}$ is the weighted market return.

2. There $i=1$ are a number of approaches in the literature addressing the country versus sector debate for global investing. For example, Rouwenhorst (1999) uses a regression-based multi-factor approach. Our new decomposition framework, on the other hand, can address the same topic with no regression assumptions.

3. Nevertheless, specific macro-driven country calls can still add value to tactical asset allocation, and can complement a sector rotation process.

4. Size buckets are deciles of companies sorted by market capitalisation. Style buckets (to appear later) refer to the 12 
style 'zones' as defined by the MSCI Global Value and Growth Index Series.

5. The focus of this European Model Portfolio is on topdown sector allocation, which is expressed through selected stocks. The portfolio snapshot we are using here is as of 30th December, 2005.

6. Detailed workings are available from the authors.

\section{References}

Goyal, A. and Santa-Clara, P. (2003) 'Idiosyncratic Risk Matters!', Journal of Finance, 58, 975-1007.
Hwang, S. and Salmon, M. (2004) 'Market Stress and Herding', Journal of Empirical Finance, 11, 585-616. Hwang, S. and Satchell, S. E. (2001) 'Properties of Crosssectional Volatility', Financial Econometric Research Centre Working Paper WP00-4, City University Business School. Hwang, S. and Satchell, S. E. (2005) 'GARCH Model with Cross-sectional Volatility; GARCHX Models', Applied Financial Economics, 15(3), 203-216.

Rouwenhorst, G. (1999) 'European Equity Markets and the EMU', Financial Analyst Journal, 55(3), 57-64.

Solnik, B. and Roulet, J. (2000) 'Dispersion as Cross-Sectional Correlation', Financial Analysts Journal, 56, 54-61. 